\title{
El deber, el poder y la gestión en el sistema educativo público costarricense
}

\author{
Duty, power and management in the Costa Rican public educational system
}

Recibido 23 agosto 2013 • Aceptado 15 noviembre 2013 • Corregido 6 diciembre 2013

\author{
Ivannia Calvo Gutiérrez? \\ Escuela de Física \\ Universidad de Costa Rica \\ San José, Costa Rica \\ ivanniacg@gmail.com
}

\begin{abstract}
Resumen. Este artículo plantea, en primer término, los retos de la Administración de la Educación costarricense actual ante las Tecnologías de la Información y la Comunicación (TIC); y la Sociedad de la Información y el Conocimiento (SIC). Posteriormente, se consideran diversos aspectos sumamente importantes, como el costo de ciertos dispositivos, algunos tipos de contextos y la sociedad red global que expone Castells; para ejecutar eficazmente un cambio significativo en una organización educativa. A la luz de estas ideas, se expone una articulación entre el deber kantiano, el poder de Castells y la gestión propuesta por Drucker; para suprimir diversas desigualdades sociales, y también confrontarla con las ideas bourdesianas sobre la Reproducción; en virtud de lo que ofrece la sociedad red global de Castells; lo cual desemboca en un análisis basado en la posición de Thomas Kuhn. Posteriormente, se muestra un panorama según el cual, el individuo que se expone a un universo de páginas electrónicas, debe ser correctamente orientado, desde la Administración de la Educación, para que utilice la información que se le ofrece, de la mejor forma posible. Finalmente, se exponen las conclusiones orientadas a la importancia sobre la indagación de posturas y/o líneas de pensamiento sumamente esenciales para la Humanidad, así como el impacto en las ideas bourdesianas, considerando la sociedad red global de Castells; y el sistema educativo público costarricense.
\end{abstract}

Palabras claves. Administración de la educación, gestión de la educación, sociedad red global, poder y gestión, deber y gestión, educación.

Abstract. This article proposes, firstly, the challenges faced by the Costa Rican Management of Education by the Information and Communications Technology (ICT); and the Society of Information and Knowledge (SIK). Subsequently, diverse and extremely important aspects are considered, such as the cost of certain devices, some types of contexts and the global network society explained by Castells; to effectively implement a significant change upon an educational organization. In light of these ideas, an articulation between Kantian duty, the power of Castells and the management proposed by Drucker is explained; in order to suppress different social inequalities, as well as to confront it with the bourdesian ideas about the reproduction; in virtue of what is offered by Castells' global network society; which leads to an analysis based on the position of Thomas Kuhn.

1 Máster en Ciencias de la Educación con énfasis en Administración Educativa de la Universidad de Costa Rica (UCR). Profesorado, Bachillerato y Licenciatura en la Enseñanza de las Ciencias de la Universidad Nacional (UNA). Actualmente se desempeña como docente de la Escuela de Física de la Universidad de Costa Rica y también como colaboradora del Programa Planetario. Asimismo es integrante de la Sección de Astronomía y Astrofísica, y del Centro de Investigaciones Espaciales (CINESPA) de la Universidad de Costa Rica. 
Afterwards, a panorama is shown according to which, the individual that is exposed to a universe of electronic pages, must be correctly oriented, starting with the Management of Education, so that this individual may utilize the information offered, in the best possible way. Finally, conclusions are drawn which are oriented towards the importance of the inquiry of postures and/or lines of thought which are exceptionally essential to humanity, such as the impact upon bourdesian ideas, considering Castells' global network society; and the Costa Rican public educational system.

Keywords. Educational administration, managment of education, global network society, power and management, duty and management, education.

\section{Introducción}

Este artículo proporciona un análisis considerando los retos de la Administración de la Educación costarricense actual ante las Tecnologías de la Información y la Comunicación (TIC), la Sociedad de la Información y el Conocimiento (SIC); así como las posturas propuestas por Kant, Drucker y Castells, las cuales pueden ser empleadas desde la Administración de la Educación, en función del factor económico (en términos de costos de ciertos dispositivos electrónicos móviles); y del tipo de individuos y sociedad a la cual se enfrenta, para minimizar no solo distintas posiciones bourdesianas, sino también para impulsar la producción de conocimiento, y disminuir en lo posible, aquellas desigualdades sociales que sesgan los distintos objetivos de las organizaciones educativas que conforman el sistema educativo público costarricense en todos los niveles. Todo esto conduce, inminentemente, a un análisis de la Administración de la Educación basado en la noción de paradigma de Thomas Kuhn.

También se hace un análisis sobre la forma en la que un individuo debe ser orientado, al estar frente a una gran cantidad de información disponible en Internet, en función de la naturaleza de la sociedad en la cual se encuentra, a nivel de la organización educativa, así como dependiendo de la globalización que caracteriza a la sociedad en general.

Este artículo expone la necesidad de que el profesional de la Administración de la Educación desarrolle no solo una constante capacitación (que tiende a ser autodidacta en la mayor cantidad de áreas posible); sino también, una cultura o disciplina dispuesta a investigar, en el amplio sentido de la palabra, sobre distintos tópicos o temáticas que de alguna manera, alteran el orden de la organización que conduce según las características que la envuelven.

\section{Retos de la Administración de la Educación costarricense actual ante las TIC y las SIC}

Los centros educativos de la sociedad costarricense actual, se enfrentan a una serie de factores que reflejan la clase social de la población que albergan en zonas urbanas y rurales, tal y como lo señala el Tercer Informe del Estado de la Educación: "Las circunstancias asociadas a recursos y características del hogar, como ingreso per cápita, clase social y clima educativo, se 
encuentran entre los factores que generan más desigualdades en el logro educativo" (CONARE, 2011 , p. 44). Esto se evidencia también, en la calidad educativa que alcance el estudiantado, que a su vez conduce a reflexionar sobre los distintos instrumentos como la coordinación y planificación administrativa, que deben implementarse desde la Administración de la Educación, para alcanzar la calidad educativa deseada y no obstruir los nobles fines y objetivos de la educación costarricense.

Estos instrumentos dependen del contexto de factores políticos, económicos, sociológicos, culturales, recursos (materiales y/o humanos); así como de su mejor aplicación en una organización educativa. En este sentido, las TIC y las SIC son una herramienta fundamental en la labor administrativa y docente; sin embargo, aún no se han explotado en su máximo apogeo, de modo que permitan disminuir ciertas disparidades sociales como la brecha digital; $y$ aumentar la conectividad de centros educativos que conlleve a desarrollar distintas estrategias que contribuyan al mejoramiento del proceso enseñanza-aprendizaje, como señala el Tercer Informe del mencionado programa (CONARE, 2011):

En el caso de las TIC, el MEP requiere la definición de una política que articule los distintos esfuerzos que hoy se dan dentro y fuera del sistema educativo, para incorporar estas tecnologías en los procesos de enseñanza-aprendizaje; al mismo tiempo, se requiere potenciar el uso deeste instrumento para que los estudiantes generen nuevos conocimientos; reducir las brechas de acceso que aún persisten en zonas urbanas y rurales, aumentar la conectividad de banda ancha de las escuelas y colegios, y reforzar la formación inicial que reciben los educadores en esta materia, ya que hoy es muy básica y restringida. (p. 45)

En este sentido, la administración de la educación tiene un reto sumamente crucial, porque no solo debe enfrentarse a problemáticas que atentan contra la calidad educativa, sino también contra aspectos culturales y sociales que interactúan inminentemente, en un complejo y diverso universo educativo. En virtud de lo anterior, el profesional que dirija una organización educativa, debe estudiar la institución y el contexto en el cual se desarrollan todos los miembros de su organización; para cumplir en lo posible con los fines y objetivos de la educación costarricense.

Es evidente que la administración de la educación actual, ante este panorama, debe replantearse muchos aspectos, temáticas y/o tópicos en materia científico-tecnológica, psicológica, pedagógica, económica, cultural, social, física y sobre todo ética; para conducir la organización de una forma dinámica y coordinadamente posible. Por esta razón se plantean dos parámetros fundamentales que el profesional de la administración de la educación innovador actual debe tomar en cuenta, uno está orientado a los costos económicos, el contexto y la sociedad; el otro, a la aplicación de ideas, posiciones y/o posturas sobre el poder, el deber y la gestión, los cuales se exponen a continuación. 


\section{2.a -Organizaciones educativas: costos, contextos y sociedad actual}

El norte hacia donde se dirija la Administración de la Educación debe considerar que actualmente, mirar el televisor, escuchar música en el reproductor, ingresar a una red social, ver el periódico en línea, ir al cine o a un concierto; implica que estamos expuestos a la publicidad en muchas áreas, una de ellas es la publicidad orientada al bajo costo de laptops, notebooks, netbooks, tabletas, celulares y muchísimos dispositivos portables. Por supuesto, que entre más altos sean los requerimientos de estos dispositivos, mayor será su precio; lo cual dependerá del dinero y el objetivo que finalmente tenga la persona que los compre.

Las virtudes de tales dispositivos aumentan, si se acompañan tanto con una conexión a Internet veloz, que permita la visualización de videos y revisar el correo, sin ningún contratiempo; como con la posibilidad de adquirir en los últimos años, equipo de cómputo a muy bajo costo, como lo señala Castro (2008), refiriéndose a equipo portátil:

La Eee PC posee varias configuraciones y sus precios varían desde los $\$ 299$ a los $\$ 499$ por lo que se convierte en una herramienta de última tecnología a muy bajo costo, masificando de esta forma el acceso. Importante destacar que el sistema operativo base es Linux y todas las herramientas que utiliza son de software libre. (p.18)

De este modo, si el profesional de la Administración de la Educación tramita la adquisición paulatina de tales dispositivos en su organización educativa, y a su vez todos los requerimientos para que funcionen de la mejor manera posible; debe garantizar un personal altamente capacitado para utilizar las herramientas de hardware y software libre; de manera que el estudiante y el docente accedan a la información que alberga Internet.

Aunado a lo anterior, debe estudiarse el contexto bajo el cual se encuentra el estudiante, el docente y la organización educativa; contexto que puede ser sociocultural, económico y político; es decir, se debe analizar, desde la Administración de la Educación la magnitud o el tamaño no solo de la brecha digital, sino también la brecha contextual a la que están expuestos los individuos de la organización educativa. De esta manera, será inminente la cantidad de estudiantes que tendrán acceso, a una sociedad conocida en términos de Castells, como la sociedad red global. Lo anterior, se esquematiza en la siguiente figura:

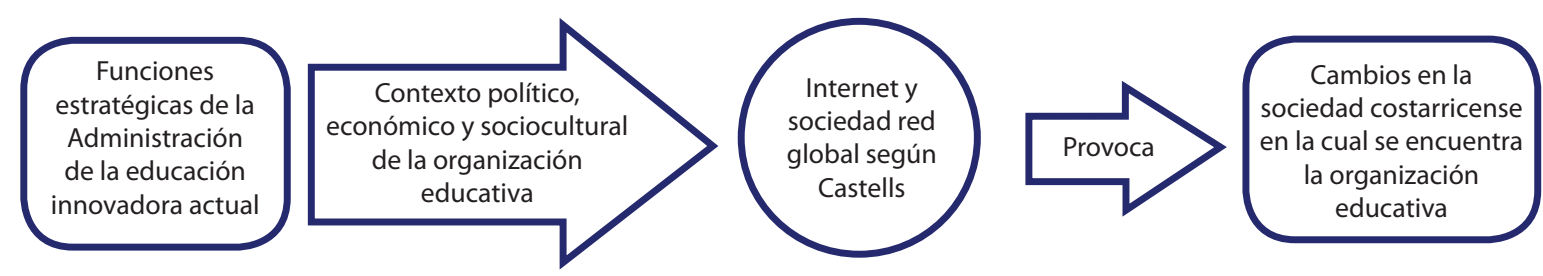

Nota: Elaboración propia, 2013.

Figura 1. Contextos, costos y sociedad red global propuesta por Castells; desde la perspectiva de la Administración de la Educación. 
De la figura anterior, se desprende que la función de la administración de la educación innovadora debe ser sumamente exhaustiva, dinámica y abierta al cambio, de modo que durante todo este proceso, articule diversas herramientas estratégicas que le permitan responder a una sociedad actual que demanda cambios profundos y de distinta naturaleza en todas las organizaciones que la componen, en función de la sociedad red global propuesta por Castells, pero debe hacerse con mayor empeño en las organizaciones educativas, porque son la base social formal sobre la cual se enseña y aprende, tal y como lo puntualiza Drucker (1999a, p. 240), cuando se refiere al libro impreso:"Esa anterior revolución tecnológica puede enseñarnos una cosa: abrazar la nueva tecnología del aprendizaje y la enseñanza será un prerrequisito del progreso nacional y cultural, y lo mismo sucederá con la competitividad económica". En síntesis, ante una revolución tecnológica las organizaciones educativas son capaces de propiciar el proceso de enseñanzaaprendizaje requerido para impulsar un futuro progreso cultural, social y económico.

En este sentido, es inevitable el impacto que provocará la restructuración de una organización, con la finalidad de adquirir y adaptarse a la tendencia que ofrece la nube de Internet en muchos campos, entre ellos, el proceso enseñanza-aprendizaje; produciendo ciertos niveles de progreso, no solo culturales o económicos, sino también enriquecimiento en la calidad y cantidad de saberes en la organización educativa, y en los individuos que la conforman, con la supervisión claro está, del profesional de la Administración de la Educación.

\section{2.b-El deber, el poder y la gestión desde la Administración de la Educación}

En el orden de la sociedad actual, es un hecho que distintos y sofisticados dispositivos portables están a disposición tanto de docentes como de estudiantes; lo cual se acentúa si se considera el bajo costo adquisitivo de dispositivos móviles, como computadoras portátiles y teléfonos celulares, entre otros. Ante este escenario, el profesional de la Administración de la Educación, es capaz de explotar el máximo potencial de estos recursos, si interioriza el deber, el poder y la gestión de la siguiente manera:

\section{El concepto del deber de Kant}

Desde esta perspectiva, es pertinente que la persona que administre una organización educativa, interiorice tanto la libertad como la facultad de decidir del ser humano, partiendo del concepto del deber del filósofo prusiano Immanuel Kant (1974, p. 47):"(...) el imperativo universal del deber podría, además, formularse de este modo: obra como si la máxima de tu acción debiera ser erigida por tu voluntad en una ley universal de la naturaleza". Es decir, el profesional de la Administración de la Educación que practica una profesión por vocación, tomó la decisión de manifestar una entrega incondicionada, y desde un ángulo kantiano, supone esfuerzos que muchas veces van contra su gusto; además, debe ser responsable y constante; sin producir daño a nadie, ni siquiera a sí mismo. Se trata, en definitiva, de que el profesional de la Administración de la Educación realice sus acciones, practicando una máxima que emana de Kant: "hágalo por usted". 
De esta manera, si desde la Administración de la Educación se estudia y se interioriza el concepto kantiano del deber y se transmite, a su vez, a todos los integrantes de la organización educativa, con especial interés a los estudiantes, se desarrollarán sin duda alguna, distintas actividades y tareas que trascenderán y se plasmarán en la sociedad global en la que está inmersa la sociedad costarricense. Trascenderán, no solo porque implica que el hacer y el actuar deben elevarse como una ley universal, tal cual lo expone Kant; sino porque a su vez, implica la explotación de otras características personales ya reseñadas por el filósofo en cuestión, como la responsabilidad, la constancia y el hecho de no hacer daño a los demás, de modo que fortalece el desarrollo de la organización educativa como un todo; y el desarrollo humano de cada uno de los sujetos que la conforman. Esta característica es sumamente pertinente, en el entorno laboral en el cual estarán los estudiantes que hoy integran nuestras organizaciones educativas.

Desde esta óptica, es evidente que el capital cultural, en términos bourdesianos, adquirido por el alumnado, está en función del contexto, el espacio y el tiempo bajo el cual se desarrolla la organización educativa; no obstante, el profesional de la Administración de la Educación, tiene la capacidad de interiorizary desarrollar un liderazgo innovador, pero considerandoal $100 \%$ el concepto del deber que propone Kant; permitiéndole así contrarrestar las condiciones que sesgan la calidad educativa de su organización, y consiguientemente fomentar el desarrollo de una identidad a nivel laboral: "El trabajar atendiendo necesidades públicas, responden a vocaciones de los funcionarios, así como, crean identidades en las labores y desempeños públicos" (Huaylupo, 2011, p. 296).

En consecuencia, si la Administración de la Educación atiende organizaciones educativas de índole público, desde la perspectiva del deber kantiano, implica que se garantizará la constancia y la disciplina en esta función pública, y al mismo tiempo la pertinencia con respecto de la eficiencia y la eficacia que emanarán de las acciones desarrolladas; y desembocarán en la optimización de la calidad de los resultados obtenidos por la organización educativa; la cual a su vez, con base en la máxima del deber kantiano fomentado desde la Administración de la Educación, le proporcionará a sus estudiantes y docentes, las herramientas intelectuales, culturales, sociales y otras; requeridas para enfrentar las distintas circunstancias de un mundo inminentemente globalizado, sobre todo aquellas que tienen la tendencia a promover la privatización de distintos bienes y servicios públicos. Se trata de adoptar el deber kantiano para fomentar de alguna manera, en nuestros estudiantes, la criticidad ante los distintos elementos a los que nos expone la sociedad costarricense actual.

\section{El concepto del poder de Castells}

Si bien una organización educativa está bajo la responsabilidad de una figura de autoridad, el concepto de poder que debe considerarse, implica que el profesional de la Administración de la Educación estudie la relación entre él y el ambiente que le rodea (contexto social, económico, 
cultural y afines); de modo que se obtenga, en lo posible, el mayor beneficio para la organización educativa. Así, en términos de Castells (2011), se puede desarrollar el poder considerándolo de la siguiente manera:

El poder es la capacidad relacional que permite a un actor social influir de forma asimétrica en las decisiones de otros actores sociales de modo que favorezcan la voluntad, los intereses y los valores del actor que tiene el poder. El poder se ejerce mediante la coacción (o la posibilidad de ejercerla) y/o mediante la construcción de significado partiendo de los discursos a través de los cuales los actores sociales guían sus acciones. Las relaciones de poder están enmarcadas por la dominación, que es el poder que reside en las instituciones de la sociedad. La capacidad relacional del poder está condicionada, pero no determinada, por la capacidad estructural de dominación. Las instituciones pueden mantener relaciones de poder que se basan en la dominación que ejercen sobre sus sujetos. (p. 33)

De la posición anterior, se puede subrayar con certeza, que las relaciones de poder se aplican a todos los sujetos participantes directos e indirectos del proceso en una organización educativa, en función de las necesidades, capacidades y objetivos de la organización, como un todo.

De este modo, considerando que algunas organizaciones educativas públicas costarricenses, visualizadas como instituciones pautadas, se caracterizan porque reciben en sus aulas, estudiantes de zonas de alto riesgo, y por lo tanto altamente marginadas; se requiere que el profesional de la Administración de la Educación, partiendo del concepto del deber kantiano señalado anteriormente, revierta la manifestación clásica de reproducción de capital cultural bourdesiano, estudiando el escenario en el que se encuentra la organización educativa. Bajo esta condición, utilizar al máximo, entre otros aspectos, los bajos costos económicos con los que se adquiere equipo de cómputo, el concepto de poder entendido como una capacidad relacional expuesta por Castells; y considerando los distintos recursos que le proporcionan las TIC; se garantiza que realmente cada miembro de la organización educativa sea capaz de empoderarse de conocimiento; empleando para ello la violencia simbólica propuesta por Bourdieu y Passeron (2001, p. 18), con un enfoque positivo: "Todo poder de violencia simbólica, o sea, todo poder que logra imponer significaciones e imponerlas como legítimas disimulando las relaciones de fuerza en que se funda su propia fuerza, añade su propia fuerza, es decir, propiamente simbólica, a esas relaciones de fuerza". Es decir, se trata de usar desde la Administración de la Educación su propia fuerza exponencialmente con un enfoque positivo, en el desarrollo de la organización educativa, para aumentar paulatinamente, los conocimientos del alumnado, e incrementar el potencial que surge de la generación de nuevos saberes, sin que en este proceso medie la prepotencia, la tiranía, la arrogancia o el despotismo; para evitar que desaparezcan las condiciones necesarias que mantienen una relación social, como lo subraya Castells (2011, p. 34):"Propongo la idea de que la pura imposición por la fuerza no es una relación social, ya que lleva a la obliteración del actor social dominado, de forma que la relación desaparece con la extinción de una de sus condiciones". 
De esta forma, es posible aplicar desde la Administración de la Educación la violencia simbólica bourdesiana que pretenda en lo posible mantener el poder, como lo expone Castells (2011), como la capacidad relacional condicionada, pero no determinada, por la capacidad estructural de dominación. Esto es un factor sumamente trascendental, porque es precisamente la clave para que el profesional de la Administración de la Educación desarrolle el liderazgo innovador, moderno y oportuno; capaz de interiorizar la importancia de articular los recursos que tiene la organización educativa, dependiendo de la población estudiantil de la que se ocupa, para alcanzar los objetivos propuestos.

Por otra parte, a pesar de que la teoría de Bourdieu explica los hechos que pueden contradecirla, tal y como lo manifiesta Dubet (1998):

La teoría de la reproducción es tan "fuerte" que explica los hechos que, a priori, la contradicen. Las excepciones estadísticas que encierran la teoría, en realidad, la confirman, al igual que la excepción confirma la regla, porque comportan ilusiones necesarias para el funcionamiento lógico del sistema. (p. 46)

Es posible estudiar, en el marco de la sociedad red global de Castells, cuál es en realidad la magnitud de las excepciones que propone Bourdieu en su Teoría de la reproducción, reseñada por Morales (2009):

(...) para Bourdieu el problema de la objetivación y estandarización de significaciones culturales particulares, hechas objetivas, está relacionada más con el proceso de reproducción de las relaciones desiguales entre las clases que conforman la sociedad. Bourdieu sostiene que en las formaciones sociales, la arbitrariedad cultural sostiene las relaciones de fuerza entre las clases sociales que constituyen una sociedad particular, a la vez que reproduce la desigualdad en la distribución del capital cultural. (p. 159)

En este sentido, no solo la Administración de la Educación sino la sociedad en su totalidad, debe confrontar la teoría de la reproducción bourdesiana con la dinámica social que ha estudiado Castells en los últimos años, y bajo la cual actúan las organizaciones educativas públicas costarricenses actuales, pues evidencia una oportunidad sumamente significativa y pertinente, para que los individuos que no son beneficiados por la excepción de Bourdieu, empiecen a surgir, usando los recursos que la sociedad red global de Castells les ofrece, de modo que se desvanezcan las nefastas desigualdades sociales a la que están sujetos, en un futuro no muy lejano.

\section{El concepto de gestión según Drucker}

Sinduda, la gestión hamarcadola forma en la queactúan distintos tipos de organizaciones, de modo que, considerando que el profesional de la Administración de la Educación tiene la libertad de decidir, y 
por lo tanto, de entregarse a la tarea de dirigir una organización educativa, y con base en tal decisión actuar; desde un ángulo kantiano, es claro que si tal decisión la articula con el concepto de gestión de Drucker (1999a, p. 59):" Proporcionar saber para averiguar en quéforma ese saber existente puede aplicarse a producir resultados es, de hecho, lo que significa gestión", los resultados conducen irrevocablemente, a producir conocimiento científico que se reflejará no solo en el progreso de la organización, sino también en el enriquecimiento de conocimiento, y por lo tanto, del capital cultural bourdesiano de los integrantes de la organización, independientemente del origen social que los caracterice.

En virtud de lo anterior, es pertinente enfatizar que, al combinar el concepto de poder de Castells, el deber de Kant y la gestión de Drucker se revierte drástica y exponencialmente la Teoría de la reproducción bourdesiana, y si se desarrolla desde un ángulo científico, se produce, para el caso particular de la Administración propiamente como ciencia, y muy específicamente en el marco educativo, la manifestación de un paradigma, tal y como lo apunta Kuhn (2006):

Concretamente, la discusión precedente ha puesto de manifiesto que aquí consideramos como revoluciones científicas aquellos episodios de desarrollo no acumulativo en los que un paradigma antiguo se ve sustituido en todo o en parte por otro nuevo incompatible con él. (p. 186)

En virtud de lo anterior, la combinación y explotación del poder como una capacidad relacional señalada por Castells, el deber kantiano y el concepto de gestión de Drucker, resulta sumamente pertinente, trascendental y espectacular; ya que desde la Administración de la educación se introduce no solo un nuevo paradigma tal y como lo expone Kuhn, sino también un "giro copernicano" como diría Kant, en el funcionamiento de una organización educativa; para que cada persona que la conforma, afiance el conocimiento necesario para entender y comprender cómo funcionan distintos componentes científicos, tecnológicos, políticos, económicos, sociales y culturales. Esto es sumamente importante para un país que no solo desea alcanzar el desarrollo, sino también entender distintos procesos o fenómenos propios de la naturaleza.

De esta manera, la trilogía deber-poder-gestión, combinada en la sociedad red global de Castells, constituye una evidencia del dinamismo que es capaz de implementar la Administración de la Educación en la actualidad, impulsando no solo su propio desarrollo como ciencia en sí misma, sino también el de las organizaciones que estudia; para producir conocimiento, como lo puntualiza Salas (2003, p. 13): “Por esta razón, se puede afirmar que la Administración Educativa se encuentra en una etapa de amplia producción teórica que pone en evidencia un estadio de madurez y desarrollo disciplinar". Así, al articular el deber, el poder y la gestión; se manifiesta inevitablemente, un punto de inflexión que trasciende la forma en la que se desarrolla la Administración de la Educación como disciplina científica. 
Lo anterior, se sintetiza en la siguiente figura:

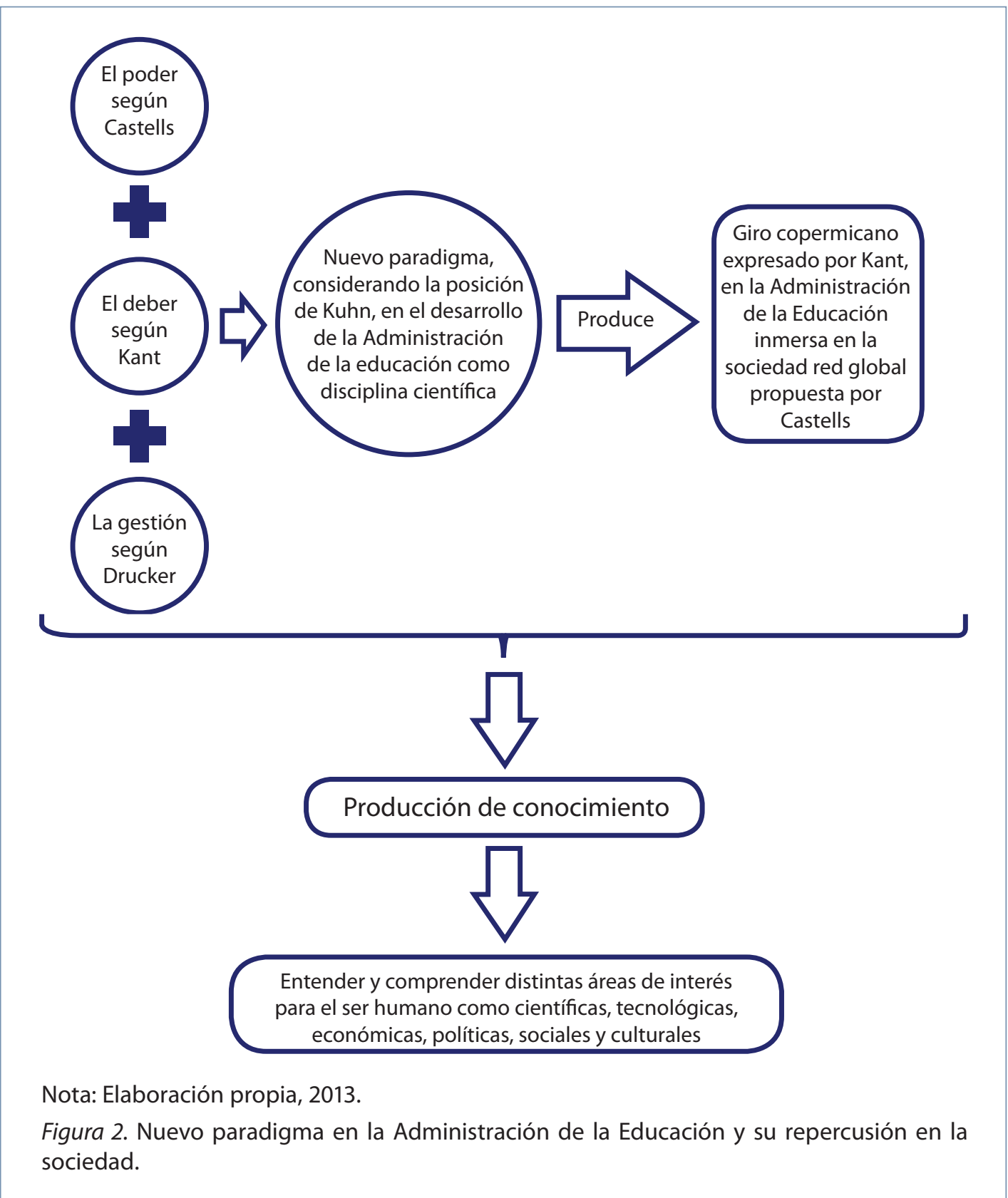


De este modo, es el profesional de la Administración de la Educación el que gesta, a través de un "giro copernicano" ya propuesto por Kant; un punto de inflexión en la producción de conocimiento en las organizaciones educativas, asumiendo su rol con una perspectiva responsable, oportuna, y a la vez flexible; abierto a los cambios, sobre todo si son repentinos; e implementando distintos mecanismos estratégicos que le permitan responder oportuna y eficazmente; a los requerimientos que le exige la organización en sí misma; para garantizar el adecuado desarrollo de la calidad, eficiencia y eficacia de la organización y de los sujetos que la componen, según los distintos contextos que envuelven al sistema educativo público costarricense, en todos los niveles. Además, se impulsará el progreso en todas las áreas posibles, en un país como el nuestro, caracterizado por estar en vías de desarrollo.

En consecuencia, la facultad y las repercusiones que tiene la Administración de la Educación como ciencia, asumida adecuadamente por la persona que conduce una organización educativa, en la sociedad costarricense actual, es fundamental en los desafíos que enfrenta diariamente, lo que representa una oportunidad para que la persona que dirige tal organización aplique el deber a su rol, desde una posición kantiana, referente a la responsabilidad y la constancia.

\section{3- Producción del conocimiento: dinámica del poder, del deber y la gestión en la sociedad red global de Castells}

En virtud de lo expuesto anteriormente, si desde la Administración de la Educación se combina el poder, el deber y la gestión; con la dinámica bajo la cual se desarrolla la sociedad red global actual (como la define Castells), es posible promover la adquisición de saberes en la organización, impulsando la capacitación del personal docente a través de plataformas educativas como coursera (www.coursera.org), en la cual se pueden estudiar cursos, donde participan algunas universidades de las más importantes y prestigiosas del mundo. Si una capacitación se planteara desde la Administración de la Educación en el sistema educativo público costarricense a través de esta página web, los resultados conllevan no solo a mejorar la calidad, la eficiencia y la eficacia de la educación misma, sino que se cumple una función propia de la organización, desde la Administración de la Educación: hacer que el conocimiento sea productivo, como lo subraya Drucker (1999a, p. 68): “La función de la organización es hacer que los saberes sean productivos; las organizaciones se han convertido en esenciales para la sociedad en todos los países desarrollados debido al paso del saber a los saberes. Cuanto más especializados sean esos saberes, más eficaces serán ellas".

De este modo, se garantizará en la población estudiantil la adquisición de conocimiento, como una de las herramientas más importantes, y la inserción en el mercado laboral en un futuro, para minimizar exponencialmente, la posición de Drucker (1999b, p. 172): "El recurso más escaso en toda organización es el personal de buen desempeño", y el punto de vista de la 
Teoría de la Reproducción propuesta por Bourdieu. Bajo este ángulo, se aplica una herramienta estratégica desde la Administración de la Educación, en todos los niveles, porque asegura la equidad necesaria que garantiza la calidad educativa, como lo manifiesta Garbanzo (2007, p. 21): “El mejoramiento de la calidad de la educación en un estado óptimo, donde la educación de calidad llegue a toda la población en igualdad de condiciones, debería implicar resolver los problemas de equidad". En este sentido, no solo se debe propiciar un equilibrio con respecto de las disparidades sociales, sino también asegurar la maquinaria humana que en un futuro constituirá la población económicamente activa y por lo tanto, la base laboral de la sociedad.

Cierto es que la sociedad costarricense posee distintas características en función dela clase social.Sin embargo, cuando una persona, independientemente de su estrato social, tiene acceso a sitios web como www.coursera.org por medio de un dispositivo móvil de bajo costo; con la mediación pedagógica idónea gestada desde la Administración de la Educación, es claro que aumenta su producción de conocimiento, de tal manera que, si bien materialmente no tenga lo básico, a nivel de saberes, es dueño de información que le permitirá desarrollar un conocimiento que nadie podrá arrebatarle.

De esta forma, debido a la naturaleza en la cual se desarrollan las organizaciones educativas actuales, así como los sujetos que la componen; Internet también ha marcado en los últimos años, no solo una fuente de información que implica el enriquecimiento de saberes tanto de cada individuo, como de la organización en su totalidad, sino que además ha provocado cambios en otras áreas o sectores políticos y económicos de nuestra sociedad, estimulando en cada persona el desarrollo de otras habilidades y destrezas intrínsecas al ser humano, unas más explotadas que otras, pero que en suma, son un indicio de la forma en la que interactúa cada actor social en la SIC, tal y como lo expone Castells (2011):

Ven televisión, están en línea, escuchan música (o la radio), leen sus SMS en el móvil y juegan con las consolas al mismo tiempo. En su uso de Internet envían correos, navegan por páginas web, leen periódicos en línea, estudian y trabajan en el mismo marco temporal. Además, no son receptores pasivos de mensajes e información. Un subgrupo significativo también produce contenidos. Remezclan vídeos y los suben, descargan y comparten música y películas y crean blogs y participan en ellos. Su uso de Internet está muy diversificado. (p. 186)

Poreso, aunado a lo anterior, no se debe negar que la población, sobre todo la más débil en términos socioeconómicos, en la actualidad tiene fácil acceso a distintos dispositivos electrónicos (como teléfonos celulares y videojuegos), y dado el bajo costo que representa tanto la compra de estos dispositivos, como el pago del servicio de Internet (aunque sea de poca velocidad), no exime a la niñez y la adolescencia costarricense del acceso a una gran variedad de información de distinta naturaleza: entretenimiento, académico o noticioso; mediante páginas electrónicas como www.youtube.com, www.secondlife.com, https:/www.coursera.org, y/o https://www.khanacademy.org/; y otros sitios de particular interés como www.bloomberg.com; que repercute de una u otra forma, entre otros, a nivel sociocultural. 
Se trata entonces de que, desde la Administración de la Educación se interiorice una cultura, en la cual se examinen todas las posibilidades que tiene el Universo de Internet, de modo que se explore indagando cómo y para qué utilizar una página o sitio web; qué causas y/o consecuencias produce la información que contiene o la forma en la cual se utiliza; considerando los objetivos que hoy tienen los medios de comunicación, tal y como lo puntualiza Castells (2011):

En el negocio de los medios de comunicación se ha producido un cambio estratégico que ha llevado de la difusión a una audiencia genérica (suponiendo su capacidad para identificarse con un mensaje homogéneo) a audiencias concretas, adaptando el mensaje al receptor. (p. 165)

Esto define cómo actúa el individuo en la sociedad red global de Castells, y se establece desde la Administración de la Educación un cambio, que determina el desarrollo de la organización educativa, y por lo tanto de la sociedad como un todo.

En consecuencia, tal y como se ilustra en la figura 3, si el profesional de la Administración de la Educación desarrolla la articulación del deber kantiano, el poder propuesto por Castells como una capacidad relacional y la gestión formulada por Drucker; en función de los bajos costos; así como el área de influencia de las TIC, marcará un punto de inflexión en la disminución de las desigualdades sociales; y a la vez aumenta la producción de conocimiento; garantizando la calidad, la eficiencia y eficacia del sistema educativo público costarricense en todos los niveles.

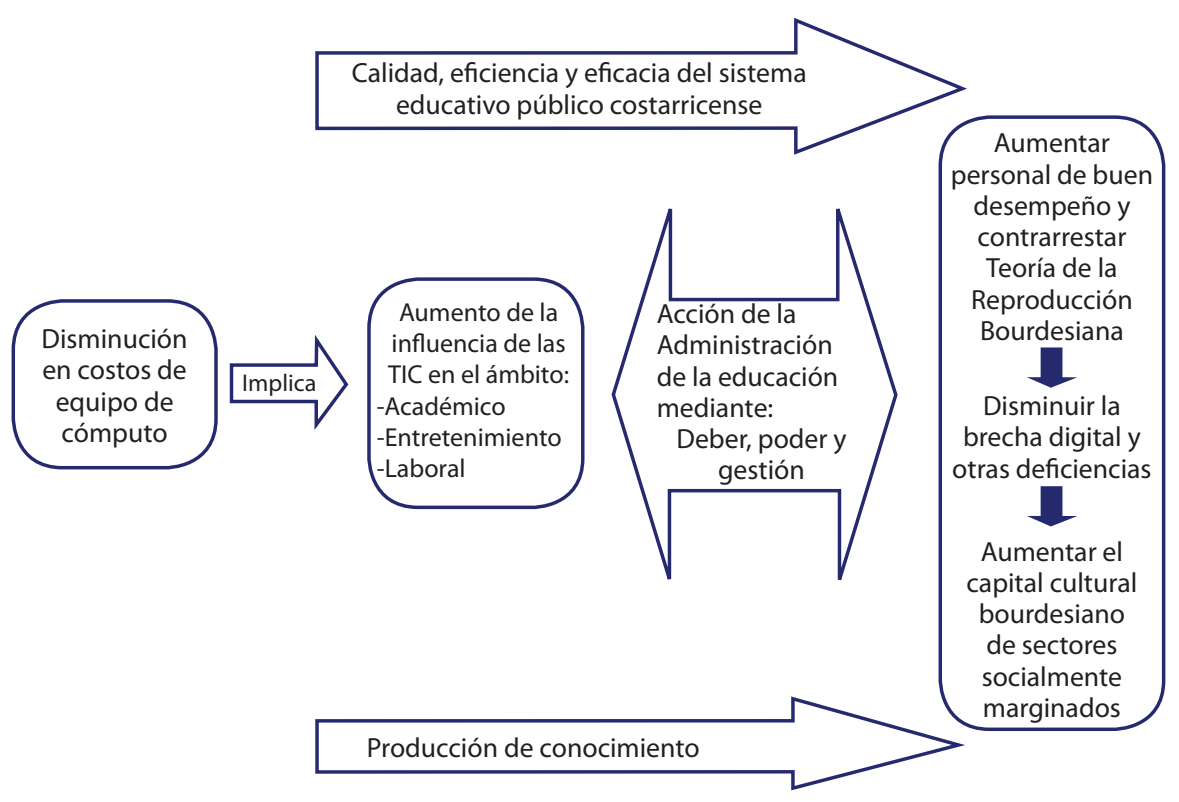

Nota: Elaboración propia, 2013.

Figura 3. El deber, el poder y la gestión en la Administración de la Educación costarricense. 


\section{Conclusión}

El profesional de la Administración de la Educación actual, debe estudiar aspectos y/o factores contextuales, políticos, sociológicos, tecnológicos, económicos y/o culturales. Sin embargo, otra de sus funciones es considerar otras corrientes o líneas de pensamiento; e implementarlas para que una organización educativa innovadora responda a las demandas de la sociedad de la cual forma parte, considerando lógicamente el contexto que lo rodea. Esto favorece el desarrollo de la organización.

Por esta razón, en este texto se analizó el concepto del deber de Kant, que considera indispensable actuar responsable y constantemente, sin hacerse daño a sí mismo ni a los demás; el del poder de Castells, entendido como una capacidad relacional, y el concepto de gestión de Drucker, referente a indagar de qué forma el conocimiento existente produce resultados.

Estos tres teóricos y conceptos, correctamente articulados desde la administración de la educación, lejos de ser una utopía, es posible considerarlos y en el mejor de los casos, aplicarlos en una organización educativa en función del contexto, lo cual desemboca en primer término en explotar al máximo desde la Administración de la Educación, el deber kantiano, para revertir en lo posible la Reproducción que propone Bourdieu; y optimizar así la utilización de las TIC con la finalidad de que no sean algunos individuos, sino la mayor cantidad de ellos, los que tengan la posibilidad de superar no solo las problemáticas y/o desigualdades sociales que enfrentan; sino también acceder en igualdad de condiciones, a la adquisición de saberes en muchas áreas; y de esta forma, la organización cumpliría su función, dotando al individuo (en la sociedad red global que describe Castells); y a la organización misma, de una de las herramientas y/o recurso más importantes, según Drucker (1999a): el saber.

Un segundo término que se debe puntualizar, es que al articular el poder, el deber y la gestión, se introduce un nuevo paradigma en el desarrollo de la Administración de la Educación como disciplina científica, que confirma su crecimiento como ciencia en sí misma, nutriéndose de posturas o aportes; para entender a su vez, otras áreas del conocimiento asociadas a un contexto marcado por la sociedad red global reseñada por Castells.

Es evidente que la investigación e indagación constante sobre los distintos factores y/o elementos sociales, políticos y/o económicos, así como de líneas de pensamiento a los cuales se expone actualmente la Administración de la Educación como ciencia, repercute no solo en el inminente dinamismo y proactividad de las organizaciones y los individuos involucrados, sino también, en el desarrollo científico que es capaz de proporcionar.

Todo lo anterior le da acceso al estudiante, desde la administración de la educación, a distinto tipo de información y conocimiento, lo cual conduce sin duda alguna, a impulsar la formación integral de personas; que en un futuro serán ciudadanos capaces de asumir una postura crítica ante la globalización, a la que se expondrán tanto en sociedad como también a nivel individual, todo esto gestado desde la administración de la educación. 
En síntesis, considerar el contexto y aquellas líneas de pensamiento que han jugado un papel fundamental en el desarrollo de la humanidad, no impide que la Administración de la Educación realice sus funciones de la mejor forma posible en aras de atacar problemas como la corrupción, la falta de ética y lealtad que hoy aquejan al sistema educativo público costarricense y la sociedad en general.

\section{Referencias}

Bourdieu, P. y Passeron, J.C. (2001). La reproducción. Elementos para una teoría del sistema de enseñanza. Madrid: Popular.

Castells, M. (2011). Comunicación y poder. Madrid: Alianza Editorial, S.A.

Castro, A. (2008). El software libre como herramienta para disminuir la brecha digital. (Ponencia para la Vigésima Cuarta Conferencia Latinoamericana de Informática, CLEl-2008).

Consejo Nacional de Rectores [CONARE]. (2011). Tercer Informe sobre el Estado de la Educación. Programa Estado de la Nación. San José, Costa Rica: CONARE.

Dubet, F. (1998). El sociólogo de la educación. Magazine Littéraire, 369, pp. 45-47. Recuperado en: http://www.pedagogica.edu.co/storage/rce/articulos/42_15contv.pdf

Drucker, P. (1999a). La sociedad poscapitalista. Buenos Aires: Sudamericana S.A.

Drucker, P. (1999b). Los desafíos de la gerencia para el siglo XXI. Bogotá: Grupo Editorial Norma.

Garbanzo V., G.M. (2007). Calidad y equidad de la educación superior pública. Aspectos por considerar en su interpretación. Revista Educación. 31(2), pp. 11-27. Recuperado en: http://revistas.ucr.ac.cr/ index.php/educacion/article/viewFile/1241/1304

Huaylupo, J., (2011). La burocracia y sus críticos en la desregulación del quehacer estatal. Revista Ciencias Económicas. 29-No 2, 287-307. Recuperado de http://www.eap.ucr.ac.cr/index.php/eapapps/descarga-de-archivos/category/12-juan-huaylupo-alcazar?download=64:la-burocracia-ysus-criticos-en-la-desregulacion-del-quehacer-estatal.

Kant, I. (1974). Fundamentación de la metafísica de las costumbres. México: Nacional.

Kuhn, T. (2006). La estructura de las revoluciones científicas. Madrid: Fondo de Cultura Económica.

Morales, L.C. (2009). Durkheim y Bourdieu: reflexiones sobre educación. Revista Reflexiones. 88 (1), pp. 155-162. Recuperado de http://www.academia.edu/340825/DURKHEIM Y BOURDIEU REFLEXIONES SOBRE EDUCACION

Salas, F. (2003). La administración educativa y su fundamentación epistemológica. Revista Educación. 27 (1), pp. 9-16. Recuperado de http://www.redalyc.org/pdf/440/44027102.pdf 